\title{
Estimation on the Generalized Half Logistic Distribution under Type-II Hybrid Censoring
}

\author{
Jung-In Seo ${ }^{a}$, Yongku Kim ${ }^{a}$, Suk-Bok Kang ${ }^{1, a}$ \\ ${ }^{a}$ Department of Statistics, Yeungnam University
}

\begin{abstract}
In this paper, we derive maximum likelihood estimators (MLEs) and approximate maximum likelihood estimators (AMLEs) of unknown parameters in a generalized half logistic distribution under Type-II hybrid censoring. We also obtain approximate confidence intervals using asymptotic variance and covariance matrices based on the MLEs and the AMLEs. As an illustration, we examine the validity of the proposed estimation using real data. Finally, we compare the proposed estimators in the sense of the mean squared error (MSE), bias, and length of the approximate confidence interval through a Monte Carlo simulation for various censoring schemes.
\end{abstract}

Keywords: Approximate maximum likelihood estimator, generalized half logistic distribution, typeII hybrid censoring.

\section{Introduction}

Half logistic distribution has been used in many reliability and survival analysis (especially when the data is censored). Inferences for the half logistic distribution have been discussed by several authors. Balakrishnan and Puthenpura (1986) introduced the best linear unbiased estimators of location and scale parameters of the half logistic distribution through linear functions of order statistics. Balakrishnan and Wong (1991) obtained AMLEs for the location parameter and the scale parameter of the half logistic distribution with Type-II right censored samples. Kang et al. (2008) derived the AMLEs and MLE of the scale parameter in a half logistic distribution based on progressively Type-II censored samples. Kang et al. (2009) proposed the AMLEs of the scale parameter in a half logistic distribution based on double hybrid censored samples. Recently, Arora et al. (2010) obtained the MLE and its asymptotic variance of the generalized half logistic distribution under Type-I progressive censoring with changing failure rates. They also provided some results including total expected waiting time in case of interval censoring schemes. Kim et al. (2011b) proposed the Bayes estimators of the shape parameter and reliability function in the generalized half logistic distribution based on progressively Type-II censored data under the various loss functions.

The cumulative distribution function (cdf) and the probability density function (pdf) of the random variable $X$ having the generalized half logistic distribution are given by

$$
F(x)=1-\left(\frac{2 e^{-\frac{x}{\sigma}}}{1+e^{-\frac{x}{\sigma}}}\right)^{\lambda}
$$

\footnotetext{
${ }^{1}$ Corresponding author: Professor, Department of Statistics, Yeungnam University, Gyeongsan 712-749, Korea.

E-mail: sbkang@yu.ac.kr
} 
and

$$
f(x)=\frac{\lambda}{\sigma}\left(\frac{2 e^{-\frac{x}{\sigma}}}{1+e^{-\frac{x}{\sigma}}}\right)^{\lambda} \frac{1}{1+e^{-\frac{x}{\sigma}}}, \quad x>0, \lambda, \sigma>0,
$$

where $\sigma$ is scale parameter and $\lambda$ is shape parameter. As a special case, if $\lambda=1$, this is the half logistic distribution. From (1.1), the $n$th moment of the generalized half logistic distribution with scale parameter $\sigma$ and shape parameter $\lambda$ can be written as

$$
\begin{aligned}
E\left(X^{n}\right) & =\int_{0}^{\infty} n x^{n-1}(1-F(x)) d x \\
& =2^{\lambda} n \int_{0}^{\infty} x^{n-1} e^{-x\left(\frac{\lambda}{\sigma}\right)}\left(1+e^{-\frac{x}{\sigma}}\right)^{-\lambda} d x \\
& =2^{\lambda} n \sum_{i=0}^{\infty}(-1)^{i}\left(\begin{array}{c}
i+\lambda-1 \\
i
\end{array}\right) \int_{0}^{\infty} x^{n-1} e^{-x\left(\frac{\lambda+i}{\sigma}\right)} d x \\
& =2^{\lambda} \sigma^{n} \Gamma(n+1) \sum_{i=0}^{\infty}(-1)^{i}\left(\begin{array}{c}
i+\lambda-1 \\
i
\end{array}\right)(\lambda+i)^{-n} .
\end{aligned}
$$

In many cases, the maximum likelihood estimation method does not provide explicit estimators based on complete and censored samples. Hence it is desirable to develop an approximation to this estimation method which would provide estimators that are explicit functions of order statistics. The approximate maximum likelihood estimation method was first developed by Balakrishnan (1989) in order to provide explicit estimators of the scale parameter in the Rayleigh distribution. Lin et al. (2006) discussed MLEs of the parameters of the log-gamma distribution based on progressively TypeII censored samples, and they derived AMLEs of the parameters and used them as initial values in the determination of the MLEs through the Newton-Raphson method. Seo and Kang (2007) derived AMLEs for Rayleigh distribution based on progressively Type-II censored data. Han and Kang (2008) derived AMLEs of the scale parameter and the location parameter in a double Rayleigh distribution based on multiply Type-II censored samples.

The most commonly used censoring schemes are Type-I and Type-II censoring schemes. In the conventional Type-I censoring scheme, the experiment continues up to a pre-specified time $T$. However, the conventional Type-II censoring scheme requires the experiment to continue until a prespecified number of failures $r \leq n$ occur without replacement. The mixture of Type-I and Type-II censoring schemes is known as a hybrid censoring scheme. Epstein (1954) first introduced Type-I hybrid censoring scheme, and considered lifetime experiments assuming that the lifetime of each unit follows an exponential distribution. Kundu (2007) developed MLEs and AMLEs for the unknown parameters under Type-I hybrid censoring. He also obtained Bayes estimators and corresponding highest posterior density credible intervals of the unknown parameters under suitable priors on the unknown parameters and using the Gibbs sampling procedure. Recently, Kang et al. (2009) derived some estimators of the scale parameter of the half triangle distribution based on Type-I hybrid censored samples. Kim et al. (2011a) suggested Bayes estimators for an exponentiated half-triangle distribution based on Type-I hybrid censoring. Under Type-I hybrid censoring scheme, the experiment is terminated at a random time $T^{*}=\min \left\{X_{r: n}, T\right\}$, where $X_{1: n}<X_{2: n}<\cdots<X_{n: n}$ and $T \in(0, \infty)$. Note that there may be few or no observed failures provided $T$ is small. Childs et al. (2003) proposed a Type-II hybrid censoring scheme to cover the disadvantage of Type-I hybrid censoring scheme. Banerjee and Kundu (2008) developed MLEs and AMLEs and discussed various Bayesian estimation methods of the two-parameter Weibull distribution under Type-II hybrid censoring. 


\section{Maximum Likelihood Estimation}

In this section, we develop MLEs of the unknown parameters in the generalized half logistic distribution under Type-II hybrid censored data. Let $X_{1: n}, X_{2: n}, \ldots, X_{n: n}$ be the order statistics of random samples $X_{1}, X_{2}, \ldots, X_{n}$. Under Type-II hybrid censoring, the experiment is terminated at a random time $T^{*}=\max \left\{X_{r: n}, T\right\}$, where $1 \leq r \leq n$ and $T \in(0, \infty)$ for fixed $r$ and $T$. In this case, we have one of the following types of observations:

Case I : $x_{1: n}<\cdots<x_{r: n}$ if $x_{r: n}>T$

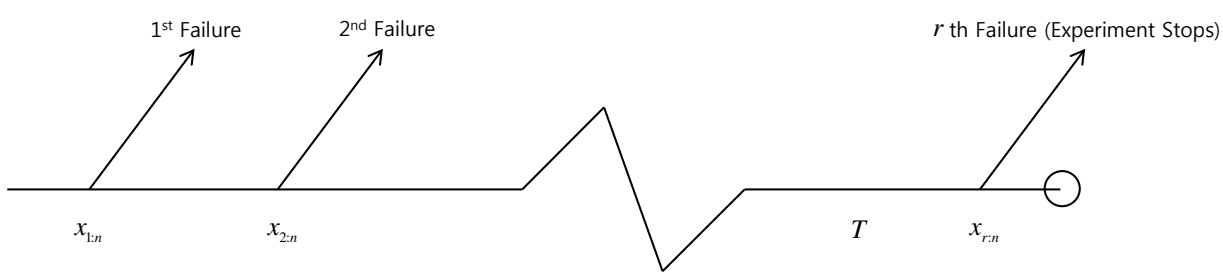

Case II : $x_{1: n}<\cdots<x_{r: n}<\cdots<x_{m: n}<T<x_{m+1: n}$ where $r \leq m<n$

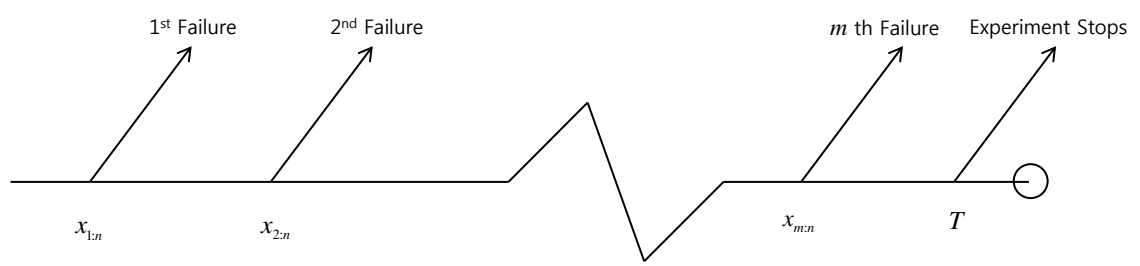

Case III : $x_{1: n}<\cdots<x_{n: n}<T$

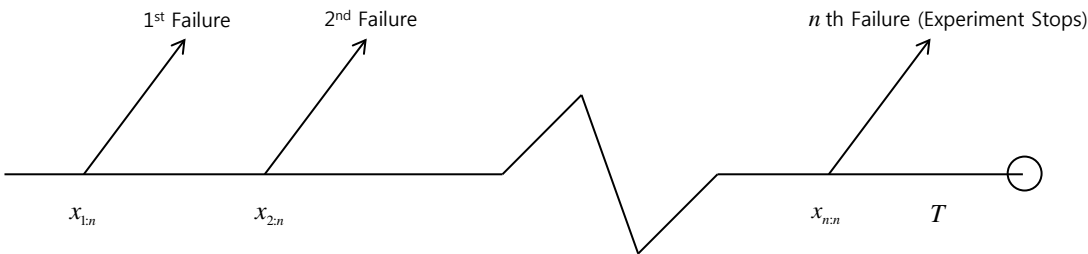

Hence, the likelihood functions for the above cases are given by

Case I : $L=\frac{n !}{(n-r) !}\left[1-F\left(x_{r: n}\right)\right]^{n-r} \prod_{i=1}^{r} f\left(x_{i: n}\right), \quad T<X_{r: n}$,

Case II : $L=\frac{n !}{(n-m) !}[1-F(T)]^{n-m} \prod_{i=1}^{m} f\left(x_{i: n}\right), \quad r \leq m<n, X_{m: n} \leq T<X_{m+1: n}$,

Case III : $L=n ! \prod_{i=1}^{n} f\left(x_{i: n}\right), \quad X_{n: n}<T$. 
Combining these cases, we can obtain the following likelihood function

$$
L=\frac{n !}{(n-s) !}[1-F(U)]^{n-s} \prod_{i=1}^{s} f\left(x_{i: n}\right),
$$

where $s$ denotes the number of failures. So $U=x_{r: n}$ if $s=r$ in Case I, and $U=T$ if $s>r$ in Case II. For simplicity, we denote order statistics $x_{1: n}, x_{2: n}, \ldots, x_{n: n}$ by $x_{1}, x_{2}, \ldots, x_{n}$.

It follows, from (1.1), (1.2), and (2.4), that

$$
L=\frac{n !}{(n-s) !}\left(\frac{\lambda}{\sigma}\right)^{s}\left(\frac{2 e^{-\frac{U}{\sigma}}}{1+e^{-\frac{U}{\sigma}}}\right)^{\lambda(n-s)} \prod_{i=1}^{s}\left(\frac{2 e^{-\frac{x_{i}}{\sigma}}}{1+e^{-\frac{x_{i}}{\sigma}}}\right)^{\lambda} \frac{1}{1+e^{-\frac{x_{i}}{\sigma}}} .
$$

The natural logarithm of the likelihood function (2.5) is given by

$$
\log L \propto s \log \lambda-s \log \sigma+\lambda(n-s) \log \left(\frac{2 e^{-\frac{U}{\sigma}}}{1+e^{-\frac{U}{\sigma}}}\right)+\lambda \sum_{i=1}^{s} \log \left(\frac{2 e^{-\frac{x_{i}}{\sigma}}}{1+e^{-\frac{x_{i}}{\sigma}}}\right)-\sum_{i=1}^{s} \log \left(1+e^{-\frac{x_{i}}{\sigma}}\right) .
$$

From (2.6), we have the likelihood equations for $\sigma$ and $\lambda$ as

$$
\begin{aligned}
\frac{\partial \log L}{\partial \sigma} & =-\frac{1}{\sigma}\left[s-\lambda(n-s) G_{1}(\sigma ; U) \frac{U}{\sigma}-\lambda \sum_{i=1}^{s} G_{1}\left(\sigma ; x_{i}\right) \frac{x_{i}}{\sigma}+\sum_{i=1}^{s}\left(1-G_{1}\left(\sigma ; x_{i}\right)\right) \frac{x_{i}}{\sigma}\right] \\
& =0
\end{aligned}
$$

and

$$
\begin{aligned}
\frac{\partial \log L}{\partial \lambda} & =\frac{s}{\lambda}+(n-s) G_{2}(\sigma ; U)+\sum_{i=1}^{s} G_{2}\left(\sigma ; x_{i}\right) \\
& =0
\end{aligned}
$$

where

$$
\begin{aligned}
& G_{1}\left(\sigma ; x_{i}\right)=\frac{1}{1+e^{-\frac{x_{i}}{\sigma}}} \\
& G_{2}\left(\sigma ; x_{i}\right)=\log \left(\frac{2 e^{-\frac{x_{i}}{\sigma}}}{1+e^{-\frac{x_{i}}{\sigma}}}\right) .
\end{aligned}
$$

Assuming that the scale parameter $\sigma$ is known, the MLE of the shape parameter $\lambda$ can be obtained as

$$
\hat{\lambda}(\sigma)=-\frac{s}{(n-s) G_{2}(\sigma ; U)+\sum_{i=1}^{s} G_{2}\left(\sigma ; x_{i}\right)} .
$$

If the scale parameter $\sigma$ is unknown, we can find the MLEs of $\sigma$ and $\lambda$ by simultaneously solving the Equation (2.7) and Equation (2.8). Unfortunately, since the Equation (2.7) cannot be solved explicitly, it may be solved by using the Newton-Raphson method that performs nonlinear optimization. The MLEs of $\sigma$ and $\lambda$ are denoted by $\hat{\sigma}$ and $\hat{\lambda}$, respectively. 


\section{Approximate Maximum Likelihood Estimation}

As mentioned earlier, because the Equation (2.7) is very complicated, it does not admit an explicit solution for $\sigma$. Therefore, we will derive some AMLEs of $\sigma$ by using the approximate likelihood equations in this section.

Let $Z_{i}=X_{i} / \sigma$ and $V=U / \sigma$. The likelihood Equation (2.7) can be written as

$$
\begin{aligned}
\frac{\partial \log L}{\partial \sigma} & =-\frac{1}{\sigma}\left[s-\lambda(n-s) \frac{1}{1+e^{-V}} V-(\lambda+1) \sum_{i=1}^{s} \frac{1}{1+e^{-z_{i}}} z_{i}+\sum_{i=1}^{s} z_{i}\right] \\
& =0
\end{aligned}
$$

Suppose $p_{i}=i /(n+1)$ and $q_{i}=1-p_{i}$ for $i=1,2, \ldots, n$. Then, $\xi_{i}=F^{-1}\left(p_{i}\right)=(-1 / \sigma) \log \left(q_{i}^{1 / \lambda} /\right.$ $\left(2-q_{i}^{1 / \lambda}\right)$ ). To obtain the approximate likelihood equation, we first approximate the following functions using Taylor series when $U=x_{r}(s=r)$ :

$$
\begin{aligned}
& \frac{1}{1+e^{-V}} \simeq \alpha_{1 r}+\beta_{1 r} z_{r}, \\
& \frac{1}{1+e^{-z_{i}}} \simeq \alpha_{1 i}+\beta_{1 i} z_{i},
\end{aligned}
$$

where

$$
\begin{aligned}
& \alpha_{1 i}=\left(1-\frac{1}{2} q_{i}^{\frac{1}{\lambda}}\right)\left(1-\frac{1}{2} q_{i}^{\frac{1}{\lambda}} \xi_{i}\right), \\
& \beta_{1 i}=\frac{1}{4} q_{i}^{\frac{1}{\lambda}}\left(2-q_{i}^{\frac{1}{\lambda}}\right) .
\end{aligned}
$$

Putting the Equation (3.2) and Equation (3.3) into the Equation (3.1), we obtain the following approximate likelihood equation

$$
\begin{aligned}
\frac{\partial \log L}{\partial \sigma} & \simeq-\frac{1}{\sigma}\left[r-\lambda(n-r)\left(\alpha_{1 r}+\beta_{1 r} z_{r}\right) z_{r}-(\lambda+1) \sum_{i=1}^{r}\left(\alpha_{1 i}+\beta_{1 i} z_{i}\right) z_{i}+\sum_{i=1}^{r} z_{i}\right] \\
& =0 .
\end{aligned}
$$

After solving the quadratic equation (3.4) for $\sigma$, we obtain AMLE of $\sigma$ as

$$
\tilde{\sigma}_{1}=\frac{A+\sqrt{A^{2}+4 r B}}{2 r},
$$

where

$$
\begin{aligned}
& A=\sum_{i}^{r} x_{i}\left((\hat{\lambda}+1) \alpha_{1 i}-1\right)+\hat{\lambda} x_{r}(n-r) \alpha_{1 r} \\
& B=\sum_{i}^{r} x_{i}^{2}(\hat{\lambda}+1) \beta_{1 i}+\hat{\lambda} x_{r}^{2}(n-r) \beta_{1 r} .
\end{aligned}
$$


If $U=T(s>r)$, instead of $\xi_{r}$, defining $\xi_{m^{*}}=-\log \left(q_{m^{*}}^{1 / \lambda} /\left(2-q_{m^{*}}^{1 / \lambda}\right)\right)$ for $p_{m^{*}}=\left(p_{m}+p_{m+1}\right) / 2$ and $q_{m^{*}}=1-p_{m^{*}}(r \leq m<n)$, the AMLE of $\sigma$ in (3.5) is written as

$$
\tilde{\sigma}_{1}=\frac{A+\sqrt{A^{2}+4 m B}}{2 m},
$$

where

$$
\begin{aligned}
& A=\sum_{i}^{m} x_{i}\left((\hat{\lambda}+1) \alpha_{1 i}-1\right)+\hat{\lambda} T(n-m) \alpha_{1 m^{*}}, \\
& B=\sum_{i}^{m} x_{i}^{2}(\hat{\lambda}+1) \beta_{1 i}+\hat{\lambda} T^{2}(n-m) \beta_{1 m^{*}} .
\end{aligned}
$$

Note that $\tilde{\sigma}_{1}$ is always positive because $\beta_{1 i}>0$. In addition, we obtain the AMLE of the shape parameter $\lambda$, denoted by $\tilde{\lambda}_{1}$, by replacing $\sigma$ with $\tilde{\sigma}_{1}$ in the Equation (2.9).

In the same way, for $U=x_{r}(s=r)$, we approximate the other functions as follows:

$$
\begin{aligned}
& \frac{1}{1+e^{-V}} V \simeq \alpha_{2 r}+\beta_{2 r} z_{r}, \\
& \frac{1}{1+e^{-z_{i}}} z_{i} \simeq \alpha_{2 i}+\beta_{2 i} z_{i},
\end{aligned}
$$

where

$$
\begin{aligned}
& \alpha_{2 i}=\frac{1}{4} q_{i}^{\frac{1}{\lambda}}\left(q_{i}^{\frac{1}{\lambda}}-2\right) \xi_{i}^{2} \\
& \beta_{2 i}=\left(1-\frac{1}{2} q_{i}^{\frac{1}{\lambda}}\right)\left(1+\frac{1}{2} q_{i}^{\frac{1}{\lambda}} \xi_{i}\right) .
\end{aligned}
$$

By using the Equation (3.7) and Equation (3.8), we obtain the following approximate likelihood equation

$$
\begin{aligned}
\frac{\partial \log L}{\partial \sigma} & \simeq-\frac{1}{\sigma}\left[r-\lambda(n-r)\left(\alpha_{2 r}+\beta_{2 r} z_{r}\right)-(\lambda+1) \sum_{i=1}^{r}\left(\alpha_{2 i}+\beta_{2 i} z_{i}\right)+\sum_{i=1}^{r} z_{i}\right] \\
& =0 .
\end{aligned}
$$

After solving the Equation (3.9) for $\sigma$, we obtain another AMLE of $\sigma$ as

$$
\tilde{\sigma}_{2}=\frac{C}{r-D}
$$

where

$$
\begin{aligned}
& C=\sum_{i=1}^{r} x_{i}\left((\hat{\lambda}+1) \beta_{2 i}-1\right)+\hat{\lambda} x_{r}(n-r) \beta_{2 r}, \\
& D=\sum_{i=1}^{r}(\hat{\lambda}+1) \alpha_{2 i}+\hat{\lambda}(n-r) \alpha_{2 r} .
\end{aligned}
$$


If $U=T(s>r)$, the AMLE of $\sigma$ in (3.10) is written as

$$
\tilde{\sigma}_{2}=\frac{C}{m-D}
$$

where

$$
\begin{aligned}
& C=\sum_{i=1}^{m} x_{i}\left((\hat{\lambda}+1) \beta_{2 i}-1\right)+\hat{\lambda} T(n-m) \beta_{2 m^{*}}, \\
& D=\sum_{i=1}^{m}(\hat{\lambda}+1) \alpha_{2 i}+\hat{\lambda}(n-m) \alpha_{2 m^{*}} .
\end{aligned}
$$

In this case, $(\lambda+1) \beta_{2 i}-1$ is positive when $\lambda>e^{-\xi_{i}}$. Therefore, we know that $\tilde{\sigma}_{2}$ is positive under the same condition. Another AMLE of $\lambda$, denoted by $\tilde{\lambda}_{2}$, is also obtained by replacing $\sigma$ with $\tilde{\sigma}_{2}$ in the Equation (2.9). Note that the estimator $\tilde{\sigma}_{2}$ is simpler than the estimator $\tilde{\sigma}_{1}$ because $\tilde{\sigma}_{2}$ is a linear combination of available order statistics.

\section{Fisher Information}

To construct confidence intervals, it is necessary to compute variance and covariance matrix of the estimators, which is given by the inverse of the Fisher information matrix.

From the Equation (2.6), we have

$$
\begin{aligned}
-\frac{\partial^{2} \log L}{\partial \sigma^{2}} & =-\frac{1}{\sigma^{2}}\left[s-\lambda(n-s) G_{3}(\sigma ; U)-\lambda \sum_{i=1}^{s} G_{3}\left(\sigma ; x_{i}\right)-\sum_{i=1}^{s} G_{4}\left(\sigma ; x_{i}\right)\right], \\
-\frac{\partial^{2} \log L}{\partial \sigma \partial \lambda} & =-\frac{1}{\sigma}\left[(n-s) G_{1}(\sigma ; U) \frac{U}{\sigma}+\sum_{i=1}^{s} G_{1}\left(\sigma ; x_{i}\right) \frac{x_{i}}{\sigma}\right], \\
-\frac{\partial^{2} \log L}{\partial \lambda^{2}} & =\frac{s}{\lambda^{2}},
\end{aligned}
$$

where

$$
\begin{aligned}
& G_{3}\left(\sigma ; x_{i}\right)=G_{1}\left(\sigma ; x_{i}\right) \frac{x_{i}}{\sigma}\left[\left(1-G_{1}\left(\sigma ; x_{i}\right)\right) \frac{x_{i}}{\sigma}+2\right], \\
& G_{4}\left(\sigma ; x_{i}\right)=\left(1-G_{1}\left(\sigma ; x_{i}\right)\right) \frac{x_{i}}{\sigma}\left(G_{1}\left(\sigma ; x_{i}\right) \frac{x_{i}}{\sigma}-2\right) .
\end{aligned}
$$

Then, by taking expectations of the (4,1), (4.2), and (4.3), we can obtain the Fisher information matrix for the MLEs $\hat{\sigma}$ and $\hat{\lambda}$ as

$$
I(\hat{\sigma}, \hat{\lambda})=E\left(\begin{array}{cc}
-\frac{\partial^{2} \log L}{\partial \sigma^{2}} & -\frac{\partial^{2} \log L}{\partial \sigma \partial \lambda} \\
-\frac{\partial^{2} \log L}{\partial \lambda \partial \sigma} & -\frac{\partial^{2} \log L}{\partial \lambda^{2}}
\end{array}\right) .
$$

In this case, it is complicated to calculate the exact expected values in the Fisher information matrix (4.4). Therefore we derive the asymptotic variance-covariance matrix by using the observed Fisher information matrix. 
The asymptotic variance-covariance matrix of the MLEs $\hat{\sigma}$ and $\hat{\lambda}$ is given by

$$
\hat{\Sigma}=\left(\begin{array}{cc}
-\frac{\partial^{2} \log L}{\partial \sigma^{2}} & -\frac{\partial^{2} \log L}{\partial \sigma \partial \lambda} \\
-\frac{\partial^{2} \log L}{\partial \lambda \partial \sigma} & -\frac{\partial^{2} \log L}{\partial \lambda^{2}}
\end{array}\right)_{(\sigma, \lambda)=(\hat{\sigma}, \hat{\lambda})}^{-1}=\left(\begin{array}{ll}
\hat{\theta}_{\sigma}^{2} & \hat{\theta}_{\sigma, \lambda} \\
\hat{\theta}_{\lambda, \sigma} & \hat{\theta}_{\lambda}^{2}
\end{array}\right) .
$$

By the asymptotic normality of the MLE, we can obtain the approximate confidence intervals of the scale parameter $\sigma$ and the shape parameter $\lambda$ to be

$$
\hat{\sigma} \pm z_{\frac{\alpha}{2}} \sqrt{\hat{\theta}_{\sigma}^{2}} \text { and } \hat{\lambda} \pm z_{\frac{\alpha}{2}} \sqrt{\hat{\theta}_{\lambda}^{2}},
$$

where $z_{\alpha / 2}$ denotes the upper $\alpha / 2$ point of the standard normal distribution.

We apply the same argument to obtain the approximate confidence intervals of the AMLEs of the scale parameter $\sigma$ and the shape parameter $\lambda$.

For $U=x_{r}$, the elements of the observed Fisher information matrix for AMLEs $\tilde{\sigma}_{1}$ and $\tilde{\lambda}_{1}$ are obtained as

$$
\begin{aligned}
\frac{\partial^{2} \log L}{\partial \sigma^{2}} \simeq & \frac{1}{\sigma^{2}}\left[r-\lambda(n-r)\left(2 \alpha_{1 r}+3 \beta_{1 r} \frac{x_{r}}{\sigma}\right) \frac{x_{r}}{\sigma}-(\lambda+1) \sum_{i=1}^{r}\left(2 \alpha_{1 i}+3 \beta_{1 i} \frac{x_{i}}{\sigma}\right) \frac{x_{i}}{\sigma}+2 \sum_{i=1}^{r} \frac{x_{i}}{\sigma}\right], \\
\frac{\partial^{2} \log L}{\partial \sigma \partial \lambda} \simeq & \frac{1}{\sigma}\left[(n-r)\left(\alpha_{1 r}+\lambda \gamma_{1 r}+\left(\beta_{1 r}+\lambda \delta_{1 r}\right) \frac{x_{r}}{\sigma}\right) \frac{x_{r}}{\sigma}\right. \\
& \left.+\sum_{i=1}^{r}\left(\alpha_{1 i}+(\lambda+1) \gamma_{1 i}+\left(\beta_{1 i}+(\lambda+1) \delta_{1 i}\right) \frac{x_{i}}{\sigma}\right) \frac{x_{i}}{\sigma}\right]
\end{aligned}
$$

where

$$
\begin{aligned}
& \gamma_{1 i}=\frac{1}{2 \lambda^{2}} q_{i}^{\frac{1}{\lambda}}\left(1-q_{i}^{\frac{1}{\lambda}}\right)\left(\log q_{i}\right) \xi_{i}, \\
& \delta_{1 i}=\frac{1}{2 \lambda^{2}} q_{i}^{\frac{1}{\lambda}}\left(q_{i}^{\frac{1}{\lambda}}-1\right)\left(\log q_{i}\right) .
\end{aligned}
$$

In the above elements, If $U=T$, then

$$
\begin{aligned}
\frac{\partial^{2} \log L}{\partial \sigma^{2}} \simeq & \frac{1}{\sigma^{2}}\left[m-\lambda(n-m)\left(2 \alpha_{1 m^{*}}+3 \beta_{1 m^{*}} \frac{T}{\sigma}\right) \frac{T}{\sigma}-(\lambda+1) \sum_{i=1}^{m}\left(2 \alpha_{1 i}+3 \beta_{1 i} \frac{x_{i}}{\sigma}\right) \frac{x_{i}}{\sigma}+2 \sum_{i=1}^{m} \frac{x_{i}}{\sigma}\right] \\
\frac{\partial^{2} \log L}{\partial \sigma \partial \lambda} \simeq & \frac{1}{\sigma}\left[(n-m)\left(\alpha_{1 m^{*}}+\lambda \gamma_{1 m^{*}}+\left(\beta_{1 m^{*}}+\lambda \delta_{1 m^{*}}\right) \frac{T}{\sigma}\right) \frac{T}{\sigma}\right. \\
& \left.+\sum_{i=1}^{m}\left(\alpha_{1 i}+(\lambda+1) \gamma_{1 i}+\left(\beta_{1 i}+(\lambda+1) \delta_{1 i}\right) \frac{x_{i}}{\sigma}\right) \frac{x_{i}}{\sigma}\right]
\end{aligned}
$$

Also, for the another AMLEs $\tilde{\sigma}_{2}$ and $\tilde{\lambda}_{2}$ when $U=x_{r}$, the elements of the observed Fisher information matrix are obtained as

$$
\frac{\partial^{2} \log L}{\partial \sigma^{2}} \simeq \frac{1}{\sigma^{2}}\left[r-\lambda(n-r)\left(\alpha_{2 r}+2 \beta_{2 r} \frac{x_{r}}{\sigma}\right)-(\lambda+1) \sum_{i=1}^{r}\left(\alpha_{2 i}+2 \beta_{2 i} \frac{x_{i}}{\sigma}\right)+2 \sum_{i=1}^{r} \frac{x_{i}}{\sigma}\right],
$$


Table 1: Failure log times to breakdown of an insulating fluid testing experiment.

\begin{tabular}{llllllll}
\hline \hline 0.270027 & 1.02245 & 1.15057 & 1.42311 & 1.54116 & 1.57898 & 1.8718 & 1.9947 \\
2.08069 & 2.11263 & 2.48989 & 3.45789 & 3.48186 & 3.52371 & 3.60305 & 4.28895 \\
\hline \hline
\end{tabular}

Table 2: Critical values and test statistic ( $p$-value) for Kolmogorov test.

\begin{tabular}{c|ccc}
\hline \hline & $(\hat{\sigma}, \hat{\lambda})$ & $\left(\tilde{\sigma}_{1}, \tilde{\lambda}_{1}\right)$ & $\left(\tilde{\sigma}_{2}, \tilde{\lambda}_{2}\right)$ \\
\hline Estimates & $(1.14089,0.68809)$ & $(1.14019,0.68758)$ & $(1.14118,0.68830)$ \\
Critical value $(\alpha=0.05)$ & 0.35390 & 0.35390 & 0.35390 \\
Test statistic $D_{n}(p$-value $)$ & $0.25036(0.226)$ & $0.25035(0.226)$ & $0.25036(0.226)$ \\
\hline \hline
\end{tabular}

$$
\frac{\partial^{2} \log L}{\partial \sigma \partial \lambda} \simeq \frac{1}{\sigma}\left[(n-r)\left(\alpha_{2 r}+\lambda \gamma_{2 r}+\left(\beta_{2 r}+\lambda \delta_{2 r}\right) \frac{x_{r}}{\sigma}\right)+\sum_{i=1}^{r}\left(\alpha_{2 i}+(\lambda+1) \gamma_{2 i}+\left(\beta_{2 i}+(\lambda+1) \delta_{2 i}\right) \frac{x_{i}}{\sigma}\right)\right],
$$

where

$$
\begin{aligned}
& \gamma_{2 i}=\frac{1}{2 \lambda^{2}} q_{i}^{\frac{1}{\lambda}}\left(\log q_{i}\right)\left(\left(1-q_{i}^{\frac{1}{\lambda}}\right) \xi_{i}-2\right) \xi_{i}, \\
& \delta_{2 i}=\frac{1}{2 \lambda^{2}} q_{i}^{\frac{1}{\lambda}}\left(\log q_{i}\right)\left(\left(q_{i}^{\frac{1}{\lambda}}-1\right) \xi_{i}+2\right) .
\end{aligned}
$$

In the above elements, if $U=T$, then

$$
\begin{aligned}
& \frac{\partial^{2} \log L}{\partial \sigma^{2}} \simeq \frac{1}{\sigma^{2}}\left[m-\lambda(n-m)\left(\alpha_{2 m^{*}}+2 \beta_{2 m^{*}} \frac{T}{\sigma}\right)-(\lambda+1) \sum_{i=1}^{m}\left(\alpha_{2 i}+2 \beta_{2 i} \frac{x_{i}}{\sigma}\right)+2 \sum_{i=1}^{m} \frac{x_{i}}{\sigma}\right] \\
& \frac{\partial^{2} \log L}{\partial \sigma \partial \lambda} \simeq \frac{1}{\sigma}\left[(n-m)\left(\alpha_{2 m^{*}}+\lambda \gamma_{2 m^{*}}+\left(\beta_{2 m^{*}}+\lambda \delta_{2 m^{*}}\right) \frac{T}{\sigma}\right)+\sum_{i=1}^{m}\left(\alpha_{2 i}+(\lambda+1) \gamma_{2 i}+\left(\beta_{2 i}+(\lambda+1) \delta_{2 i}\right) \frac{x_{i}}{\sigma}\right)\right] .
\end{aligned}
$$

Note that we use the Equation (4.3) as a value of the second partial derivative of the logarithm of the likelihood function for the shape parameter $\lambda$ in the observed Fisher information matrix for the two types of AMLEs.

\section{Illustrative Example}

In this section, we present an example to illustrate our estimation methods and assess the performance of the estimators discussed in the previous sections.

\subsection{Real data}

We consider the failure log times to breakdown of an insulating fluid testing experiment (Nelson, 1982 and see Table 1). This data has been utilized by many authors, such as Balakrishnan and Kannan (2001), Balakrishnan et al. (2004), and Kim et al. (2011b). We apply the Kolmogorov test to examine whether the data follow a generalized half logistic distribution with the unknown scale and shape parameters. We first calculate the MLEs and the AMLEs of the unknown parameters $\sigma$ and $\lambda$ for uncensored data; subsequently, using these results, we create the critical values for the Kolmogorov test statistic for a generalized half logistic distribution by Monte Carlo method. Finally, we obtain the values of the Kolmogorov test statistics $D_{n}$ and associated $p$-values. These values are given in Table 2. Since all the critical values at the significance level of $\alpha=0.05$ exceed the corresponding values of the test statistics, we can conclude that the data follow a generalized half logistic distribution. 
Table 3: MLEs, AMLEs, and their standard deviations (SD) for real data.

\begin{tabular}{c|ccc}
\hline & $\hat{\sigma}$ & $\tilde{\sigma}_{1}$ & $\tilde{\sigma}_{2}$ \\
\hline Estimates & 0.83449 & 0.84257 & 0.84059 \\
SD & 0.12050 & 0.11987 & 0.11946 \\
\hline & $\hat{\lambda}$ & $\tilde{\lambda}_{1}$ & $\tilde{\lambda}_{2}$ \\
\hline Estimates & 0.40709 & 0.41278 & 0.41160 \\
SD & 0.12744 & 0.12617 & 0.12530 \\
\hline \hline
\end{tabular}

Table 4: The mean squared errors and biases for the estimators of the scale parameter $\sigma$ and the shape parameter $\lambda$.

\begin{tabular}{|c|c|c|c|c|}
\hline \multicolumn{5}{|c|}{ MSE(bias) } \\
\hline$T$ & $r(n=30)$ & $\hat{\sigma}$ & $\tilde{\sigma}_{1}$ & $\tilde{\sigma}_{2}$ \\
\hline \multirow{2}{*}{3.0} & 26 & $0.03388(0.02111)$ & $0.02838(0.00997)$ & $0.02596(-0.07042)$ \\
\hline & 28 & $0.03265(0.02009)$ & $0.02796(0.00951)$ & $0.02595(-0.07044)$ \\
\hline \multirow{2}{*}{3.5} & 26 & $0.03182(0.01162)$ & $0.02767(0.00521)$ & $0.02580(-0.07174)$ \\
\hline & 28 & $0.03161(0.01150)$ & $0.02761(0.00516)$ & $0.02580(-0.07174)$ \\
\hline \multirow{2}{*}{4.0} & 26 & $0.03013(0.00550)$ & $0.02697(0.00207)$ & $0.02570(-0.07219)$ \\
\hline & 28 & $0.02995(0.00541)$ & $0.02691(0.00203)$ & $0.02570(-0.07219)$ \\
\hline$T$ & $r(n=40)$ & $\hat{\sigma}$ & $\tilde{\sigma}_{1}$ & $\tilde{\sigma}_{2}$ \\
\hline \multirow{2}{*}{3.0} & 36 & $0.02447(0.01864)$ & $0.02117(0.00917)$ & $0.02082(-0.06910)$ \\
\hline & 38 & $0.02327(0.01717)$ & $0.02072(0.00849)$ & $0.02079(-0.06917)$ \\
\hline \multirow{2}{*}{3.5} & 36 & $0.02334(0.01074)$ & $0.02075(0.00512)$ & $0.02071(-0.07057)$ \\
\hline & 38 & $0.02311(0.01053)$ & $0.02067(0.00502)$ & $0.02071(-0.07057)$ \\
\hline \multirow{2}{*}{4.0} & 36 & $0.02198(0.00432)$ & $0.02018(0.00172)$ & $0.02065(-0.07118)$ \\
\hline & 38 & $0.02180(0.00419)$ & $0.02012(0.00166)$ & $0.02065(-0.07119)$ \\
\hline$T$ & $r(n=30)$ & $\hat{\lambda}$ & $\tilde{\lambda}_{1}$ & $\tilde{\lambda}_{2}$ \\
\hline \multirow{2}{*}{3.0} & 26 & $0.05466(0.07430)$ & $0.02181(0.04685)$ & $0.02567(-0.14376)$ \\
\hline & 28 & $0.04974(0.07129)$ & $0.02012(0.04508)$ & $0.02558(-0.14455)$ \\
\hline \multirow{2}{*}{3.5} & 26 & $0.04103(0.04212)$ & $0.01656(0.02623)$ & $0.02769(-0.15507)$ \\
\hline & 28 & $0.04015(0.04175)$ & $0.01627(0.02601)$ & $0.02767(-0.15516)$ \\
\hline \multirow{2}{*}{4.0} & 26 & $0.03182(0.02191)$ & $0.01280(0.01318)$ & $0.02870(-0.16120)$ \\
\hline & 28 & $0.03113(0.02167)$ & $0.01257(0.01305)$ & $0.02868(-0.16126)$ \\
\hline$T$ & $r(n=40)$ & $\hat{\lambda}$ & $\tilde{\lambda}_{1}$ & $\tilde{\lambda}_{2}$ \\
\hline \multirow{2}{*}{3.0} & 36 & $0.03294(0.06535)$ & $0.01358(0.04225)$ & $0.02374(-0.14330)$ \\
\hline & 38 & $0.02787(0.06094)$ & $0.01172(0.03957)$ & $0.02373(-0.14452)$ \\
\hline \multirow{2}{*}{3.5} & 36 & $0.02501(0.03880)$ & $0.01049(0.02506)$ & $0.02594(-0.15336)$ \\
\hline & 38 & $0.02428(0.03816)$ & $0.01021(0.02467)$ & $0.02594(-0.15353)$ \\
\hline \multirow{2}{*}{4.0} & 36 & $0.01717(0.01777)$ & $0.00725(0.01132)$ & $0.02721(-0.15991)$ \\
\hline & 38 & $0.01644(0.01741)$ & $0.00700(0.01111)$ & $0.02720(-0.16000)$ \\
\hline
\end{tabular}

In this example, we consider censoring scheme $T=2.5$ and $r=10$ (that is, $s=11$ and $U=2.5$ ). Using the formulas in Section 2 and Section 3, we obtain the MLEs and the AMLEs for the scale parameter $\sigma$ and the shape parameter $\lambda$. Standard deviations of these estimators are computed using the observed Fisher information matrices that were illustrated in the Section 4. These values are given in Table 3. From the table, we can see that all the estimators have nearly the same values for considered scheme. In addition, it is observed that the standard deviation of $\tilde{\sigma}_{2}$ has the smallest value for the scale parameter $\sigma$ and the standard deviation of $\tilde{\lambda}_{2}$ has the smallest value for the shape parameter $\lambda$.

\subsection{Simulation study}

To compare the performance of the proposed estimators, we simulate the MSEs, the biases, and the 
Table 5: The average lengths of the 95\%(90\%) approximate confidence intervals of the MLEs and AMLEs.

\begin{tabular}{|c|c|c|c|c|}
\hline \multicolumn{5}{|c|}{$95 \%(90 \%)$} \\
\hline$T$ & $r(n=30)$ & $\hat{\sigma}$ & $\tilde{\sigma}_{1}$ & $\tilde{\sigma}_{2}$ \\
\hline \multirow{2}{*}{3.0} & 26 & $0.76425(0.64472)$ & $0.71108(0.59986)$ & $0.62845(0.53016)$ \\
\hline & 28 & $0.75225(0.63459)$ & $0.70662(0.59610)$ & $0.62827(0.53000)$ \\
\hline \multirow{2}{*}{3.5} & 26 & $0.74657(0.62980)$ & $0.70416(0.59402)$ & $0.62418(0.52655)$ \\
\hline & 28 & $0.74448(0.62804)$ & $0.70354(0.59350)$ & $0.62425(0.52661)$ \\
\hline \multirow{2}{*}{4.0} & 26 & $0.73028(0.61605)$ & $0.69690(0.58790)$ & $0.62219(0.52487)$ \\
\hline & 28 & $0.72838(0.61445)$ & $0.69626(0.58736)$ & $0.62215(0.52484)$ \\
\hline$T$ & $r(n=40)$ & $\hat{\sigma}$ & $\tilde{\sigma}_{1}$ & $\tilde{\sigma}_{2}$ \\
\hline \multirow{2}{*}{3.0} & 36 & $0.66514(0.56110)$ & $0.62941(0.53097)$ & $0.56524(0.47683)$ \\
\hline & 38 & $0.65181(0.54986)$ & $0.62411(0.52650)$ & $0.56469(0.47637)$ \\
\hline \multirow{2}{*}{3.5} & 36 & $0.65478(0.55236)$ & $0.62505(0.52728)$ & $0.56102(0.47327)$ \\
\hline & 38 & $0.65214(0.55014)$ & $0.62407(0.52646)$ & $0.56104(0.47329)$ \\
\hline \multirow{2}{*}{4.0} & 36 & $0.63991(0.53982)$ & $0.61834(0.52162)$ & $0.55902(0.47159)$ \\
\hline & 38 & $0.63780(0.53804)$ & $0.61763(0.52102)$ & $0.55900(0.47156)$ \\
\hline$T$ & $r(n=30)$ & $\hat{\lambda}$ & $\tilde{\lambda}_{1}$ & $\tilde{\lambda}_{2}$ \\
\hline \multirow{2}{*}{3.0} & 26 & $0.90709(0.76521)$ & $0.61145(0.51581)$ & $0.38931(0.32842)$ \\
\hline & 28 & $0.86872(0.73284)$ & $0.59207(0.49946)$ & $0.38302(0.32311)$ \\
\hline \multirow{2}{*}{3.5} & 26 & $0.82005(0.69179)$ & $0.56296(0.47490)$ & $0.36179(0.30520)$ \\
\hline & 28 & $0.81218(0.68515)$ & $0.55916(0.47170)$ & $0.36068(0.30426)$ \\
\hline \multirow{2}{*}{4.0} & 26 & $0.74311(0.62688)$ & $0.51722(0.43632)$ & $0.34164(0.28821)$ \\
\hline & 28 & $0.73605(0.62092)$ & $0.51395(0.43356)$ & $0.34079(0.28749)$ \\
\hline$T$ & $r(n=40)$ & $\hat{\lambda}$ & $\tilde{\lambda}_{1}$ & $\tilde{\lambda}_{2}$ \\
\hline \multirow{2}{*}{3.0} & 36 & $0.71524(0.60337)$ & $0.50484(0.42588)$ & $0.35256(0.29742)$ \\
\hline & 38 & $0.66554(0.56144)$ & $0.47949(0.40449)$ & $0.34458(0.29069)$ \\
\hline \multirow{2}{*}{3.5} & 36 & $0.65806(0.55513)$ & $0.47477(0.40051)$ & $0.33506(0.28266)$ \\
\hline & 38 & $0.65004(0.54837)$ & $0.47070(0.39707)$ & $0.33385(0.28163)$ \\
\hline \multirow{2}{*}{4.0} & 36 & $0.57609(0.48599)$ & $0.42869(0.36164)$ & $0.31692(0.26735)$ \\
\hline & 38 & $0.56649(0.47788)$ & $0.42433(0.35796)$ & $0.31584(0.26644)$ \\
\hline
\end{tabular}

average lengths of the approximate confidence intervals of all derived estimators through a Monte Carlo simulation method. By modifying the original algorithm presented in Balakrishnan and Sandhu (1995), we generate Type-II hybrid censored data from the standard generalized half logistic distribution when the shape parameter $\lambda=2$. The modified algorithm has the following steps:

(1) Generate $n$ independent $U(0,1)$ random variables $W_{1}, W_{2}, \ldots, W_{n}$.

(2) Set $V_{i}=W_{i}^{1 / i}$ for $i=1,2, \ldots, n$.

(3) Set $U_{i}=1-\left(V_{n} V_{n-1} \cdots V_{n-i+1}\right)$ for $i=1,2, \ldots, n$. Then $U_{1}<U_{2}<\cdots<U_{n}$ are data of size $n$ from $U(0,1)$.

(4) For $\lambda=2$, set $X_{i}=F^{-1}\left(U_{i}\right)=-\log \left[\left(1-U_{i}\right)_{i}^{1 / \lambda} /\left\{2-\left(1-U_{i}\right)_{i}^{1 / \lambda}\right\}\right], i=1,2, \ldots, n$. Then $X_{1}<X_{2}<$ $\cdots<X_{n}$ are data of size $n$ from the standard generalized half logistic distribution when the shape parameter $\lambda=2$.

(5) Choose Type-II hybrid censoring scheme $T$ and $r$.

For sample size $n=30$ and 40, we calculate the MLEs and AMLEs and obtain the corresponding $95 \%$ approximate confidence intervals and $90 \%$ approximate confidence intervals using the data obtained above. By repeating this procedure 10,000 times, we obtain the MSEs and the biases of the 
MLEs and AMLEs. In addition, we obtain the average lengths of the $95 \%$ and $90 \%$ approximate confidence intervals of the MLEs and AMLEs for $\sigma$ and $\lambda$. These values are given in Table 4 and Table 5. From the tables, we can see that the MSEs decrease as $r$ increases for the considered cases. Except for the AMLE $\tilde{\lambda}_{2}$, the MSEs decrease as $T$ increases; in addition, the AMLEs are more efficient than their corresponding MLEs for both of the unknown parameters $\sigma$ and $\lambda$. For the scale parameter $\sigma$, the AMLE $\tilde{\sigma}_{2}$ is generally superior to the AMLE $\tilde{\sigma}_{1}$. For the shape parameter $\lambda$, the AMLE $\tilde{\lambda}_{1}$ show an overall better performance than the another AMLE $\tilde{\lambda}_{2}$. Furthermore, the length of the $95 \%$ approximate confidence intervals of the AMLEs are smaller than that of the $95 \%$ approximate confidence intervals of the MLEs for both of the unknown parameters $\sigma$ and $\lambda$, especially in the AMLEs $\tilde{\sigma}_{2}$ and $\tilde{\lambda}_{2}$. We have the same results for the $90 \%$ approximate confidence intervals.

\section{Concluding Remarks}

This paper develops the MLEs of the scale and shape parameters in a generalized half logistic distribution under Type-II hybrid censoring. The MLE of the scale parameter cannot be explicitly solved and we therefore propose two types of AMLEs as an alternative. We can obtain the AMLEs by solving the approximate likelihood equations, and their standard deviations by using the observed Fisher information matrices. We compared these estimators through a Monte Carlo simulation. Our results show that the AMLEs are superior to their corresponding MLEs for the considered cases. That is, the AMLEs take a closed form, and besides they outperform the corresponding MLEs. Therefore we can recommend the use of the AMLEs.

\section{References}

Arora, S. H., Bhimani, G. C. and Patel, M. N. (2010). Some results on maximum likelihood estimators of parameters of generalized half logistic distribution under Type-I progressive censoring with changing, International Journal of Contemporary Mathematical Sciences, 5, 685-698.

Balakrishnan, N. (1989). Approximate MLE of the scale parameter of the Rayleigh distribution with censoring, IEEE Transactions on Reliability, 38, 355-357.

Balakrishnan, N. and Kannan, N. (2001). Point and interval estimation for the logistic distribution based on progressively Type-II censored samples, in Handbook of Statistics, Balakrishnan, N. and Rao, C. R., Eds. 20, 431-456.

Balakrishnan, N., Kannan, N., Lin, C. T. and Wu, S. J. S. (2004). Inference for the extreme value distribution under progressively Type-II censoring, Journal of Statistical Computation and Simulation, 74, 25-45.

Balakrishnan, N. and Puthenpura, N. (1986). Best linear unbiased estimators of location and scale parameters of the half logistic distribution, Journal of Statistics and Computer Simulation, 25, 193-204.

Balakrishnan, N. and Sandhu, R. A. (1995). A simple simulational algorithm for generating progressively Type-II censored samples, The American Statistician, 49, 229-230.

Balakrishnan, N. and Wong, K. H. T. (1991). Approximate MLEs for the location and scale parameters of the half-logistic distribution with Type-II right censoring, IEEE Transactions on Reliability, 40, 140-145.

Banerjee, A. and Kundu, D. (2008). Inference based on Type-II hybrid censored data from a Weibull Distribution, IEEE Transactions on Reliability, 57, 369-378.

Childs, A., Chandrasekhar, B., Balakrishnan, N. and Kundu, D. (2003). Exact likelihood inference based on Type-I and Type-II hybrid censored samples from the exponential distribution, Annals 
of the Institute of Statistical Mathematics, 55, 319-330.

Epstein, B. (1954). Truncated life tests in the exponential case, Annals of the Institute of Statistical Mathematics, 25, 555-564.

Han, J. T. and Kang, S. B. (2008). Estimation for the double Rayleigh distribution based on multiply Type-II censored samples, Communications of the Korean Statistical Society, 15, 367-378.

Kang, S. B., Cho, Y. S. and Han, J. T. (2008). Estimation for the half logistic distribution under progressively Type-II censoring, Communications of the Korean Statistical Society, 15, 815-823.

Kang, S. B., Cho, Y. S. and Han, J. T. (2009). Estimation for the half logistic distribution based on double hybrid censored samples, Communications of the Korean Statistical Society, 16, 10551066.

Kang, S. B., Cho, Y. S. and Han, J. T. (2009). Estimation for the half triangle distribution based on Type-I Hybrid Censored Sample, Journal of the Korean Data $\mathcal{F}$ Information Science Society, 20, 961-969.

Kim, Y. K., Kang, S. B. and Seo, J. I. (2011a). Bayesian estimations on the exponentiated half triangle distribution under Type-I hybrid censoring, Journal of the Korean Data \& Information Science Society, 22, 565-574.

Kim, Y. K., Kang, S. B. and Seo, J. I. (2011b). Bayesian estimation in the generalized half logistic distribution under progressively Type-II censoring, Journal of the Korean Data \& Information Science Society, 22, 977-987.

Kundu, D. (2007). On hybrid censored Weibull distribution, Journal of Statistical Planning and Inference, 137, 2127-2142.

Lin, C. T., Wu, S. J. S. and Balakrishnan, N. (2006). Inference for log-gamma distribution based on progressively Type-II censored data, Communication in Statistics-Theory and Methods, 35, 1271-1292.

Nelson, W. B. (1982). Applied Life Data Analysis, John Willey \& Sons, New York.

Seo, E. H. and Kang, S. B. (2007). AMLEs for Rayleigh distribution based on progressively Type-II censored data, The Korean Communications in Statistics, 14, 329-344. 